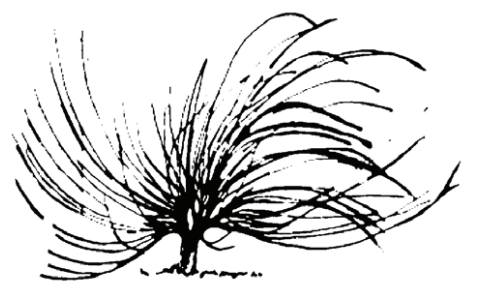

\title{
Tejiendo Procesos de Aprendiencia por Medio de las TIC: UNA Experiencia con Docentes de la Zona Rural
}

\author{
Dilia Colindres Molina ${ }^{1}$ \\ Universidad Nacional \\ Heredia, Costa Rica \\ diliacolindres@gmail.com \\ Marvin Fernández Valverde ${ }^{3}$ \\ fermarvin@gmail.com \\ Universidad Nacional \\ Heredia, Costa Rica
}

\author{
Elionay Quirós Menéses ${ }^{2}$ \\ Universidad Nacional \\ Heredia, Costa Rica \\ equiroster@gmail.com
}

Ana Azofeifa Lizano
Universidad Nacional
Heredia, Costa Rica
anaazofeifa@gmail.com

\begin{abstract}
Resumen
En este artículo se exponen los resultados de un taller de capacitación desarrollado con un grupo de docentes de secundaria y primaria de Nandayure de Nicoya, en la provincia de Guanacaste. Sus participantes elaboraron materiales y recursos didácticos con apoyo de las tecnologías de la información y comunicación (TIC), posteriormente, las utilizaron en el desarrollo de sus clases. El proceso inició
\end{abstract}

Recibido: 5 de febrero de 2014 - Aprobado: 18 de agosto de 2014

1 Docente de la División de Educología (CIDE), UNA. Especialista en pedagogía, educación física, administración educativa y mediación pedagógica.

2 Docente e investigador-extensionista de la División de Educología (CIDE), UNA. Graduado en Ciencia de la Educación I y II Ciclo con Enfasis en Educación Rural. Actualmente labora en el Proyecto Esperanza Jóven de la División de Educología.

3 Académico investigador en la División de Educología (CIDE), UNA. Profesor de informática educativa del PRONIE-FOD-MEP. Máster en Tecnología e Informática Educativa (UNA. Máster en Gestión Educativa con Énfasis en Liderazgo (UNA). Licenciado en Estudios Latinoamericanos (UNA).

4 Docente de la División de Educología (CIDE), UNA. Especialista en educación física y gestión educativa con énfasis en liderazgo. 
con un diagnóstico de las necesidades de capacitación de los docentes de esa zona. Los investigadores se reunían semanalmente para planificar, seleccionar e integrar las tecnologías de acuerdo con las especialidades del personal docente participante. Luego, en las capacitaciones, se llevó a cabo una coevaluación para conocer la opinión del profesorado acerca del trabajo realizado en el proceso de aprendizaje y, de esta manera, identificar fortalezas y oportunidades de mejoramiento en los procesos de mediación pedagógica de docentes de las zonas rurales. La condición actual y compleja que enfrenta la educación requiere en las aulas de trasformaciones en la mediación pedagógica. Por esto, se abordó la necesidad de actualizar al profesorado sobre diversas temáticas, en las cuales, por medio de las TIC, se integraron acciones entre algunas especialidades. De esta manera, se proponen herramientas que facilitan el proceso y motiven el proceso de enseñanza y aprendizaje.

Palabras clave: mediación, currículo integrado, tecnologías de la información (TIC), educación rural, aprendizaje

\begin{abstract}
This article describes the results of a workshop developed with a group of elementary and high school teachers from the rural zone of Nicoya, where they had the opportunity to elaborate materials and teaching resources supported by information and communication technologies (ICT's) that they later used in the development of their classes according to their different specialties. The process began with a diagnosis to understand the necessary training topics for the teachers from this zone. Then, the researchers met week by week in order to integrate technologies with the topics of the courses mentioned previously. Later, when the workshops were developed, the opinion of the teachers regarding the work done by the facilitators was evaluated, and the learning process was peer evaluated. In this way, the strengths and opportunities for improvement in the pedagogic mediation processes were identified. This methodology allowed for transformations to begin the learning processes, improving and positively impacting the student body, teachers, and the traditional curriculum that has always been used and to begin taking actions conducive to curriculum integration mediated by technologies.
\end{abstract}


Keywords: mediation, integrated curriculum, information and communication technologies (ICT's), rural education, learning

\section{Introducción}

$\mathrm{E}$

n este artículo se exponen los resultados de un taller de capacitación, desarrollado con un grupo de docentes de secundaria y primaria de Nandayure de Nicoya, en la provincia de Guanacaste.

El propósito de esta experiencia consistió en fortalecer los procesos de integración del quehacer docente de esta zona rural, en el uso de las tecnologías (TIC), para desarrollar espacios de formación y actualización, con el objetivo de fortalecer el nivel de educación de estas zonas.

El desarrollo de las TIC es un fenómeno que está cambiando nuestro sistema educativo. A pesar de su fuerte presencia, no deja de ser realmente difícil su implementación plena en los contextos escolares, donde uno de los factores limitantes claves es la formación inicial y permanente del personal docente (Salmerón, 2011). El uso apropiado y creativo de las TIC permite al grupo docente, transformar, radicalmente, sus modelos didácticos en todas las áreas del currículo; a medida que se apropia de ellas, cambia su papel tradicional y también se derrumban los límites tradicionalmente impuestos al currículo, permitiendo que las diversas disciplinas se integren e interactúen en la realización de tareas y proyectos (Salmerón, 2011).

En la sociedad actual, las tecnologías de la información y la comunicación (TIC) constituyen un gran reto como medio transformador de los procesos de aprendizajes en las zonas rurales. Estas posibilitan cambios que permiten ofrecer una educación de calidad y actualizada, a través de ambientes de aprendizaje diferentes, creativos e innovadores. Sin embargo, pese a las necesidades en el uso de las TIC, no se puede dejar de lado, como resalta Gutiérrez (2007), que el énfasis debería estar en la reflexión sobre su impacto en nuestra sociedad, sobre su influencia en nuestras vidas, sus ventajas e inconvenientes, donde el de manejo de equipos y programas vendrán por añadidura.

\section{Las tecnologías de la información y la comunicación (TIC)}

El término TIC se refiere al conjunto de instrumentos, teorías y técnicas que mejoran la capacidad del ser humano para interactuar, 
con el fin de obtener y divulgar el conocimiento entre sujetos que manifiestan la voluntad de comunicarse (Monje, 2008). A través de ellas, se logra el rápido y fácil acceso a la información, a los recursos y los servicios prestados por computadores. Han penetrado y transformado todas las estructuras económicas, sociales y culturales, con un alcance planetario global (Gómez, 2004).

Entre estas estructuras se encuentra el sistema educativo. De ello se deriva la creciente demanda a la actualización de docentes, en el empleo de estas tecnologías, para impulsar el cambio hacia un nuevo paradigma educativo más personalizado y centrado en la actividad de los estudiantes, que favorezca el logro de una escuela más eficaz e inclusiva (Marqués, 2008).

Gómez (2004, citado en Alonso y Zabatta, 2012) considera tres maneras de insertar las TIC en el sistema educativo: (a) como objeto de aprendizaje: permite familiarizarse con el manejo del computador y adquirir las competencias necesarias para usarlo como herramienta de apoyo en sus estudios y en sus labores dentro del ámbito profesional; (b) como medio para aprender: se transforman en una herramienta al servicio del autoaprendizaje y de la formación a distancia; y (c) como apoyo al aprendizaje: se integran a los procesos pedagógicos.

\section{Integración curricular}

La integración curricular permite una organización de contenidos temáticos con el propósito de propiciar y promover un aprendizaje significativo, funcional, auténtico y relacionado entre sí mismo.

En un currículo integrado se deben tomar en consideración los conocimientos previos del estudiantado, experiencias, actitudes y hábitos; incluso existe la posibilidad de que el alumnado mismo planifique algunos temas de interés. En otros casos, de acuerdo con sus necesidades e intereses, puede planear junto con sus docentes. Además, debe tratar de seguir una organización y secuencia lógica de los temas (Cintrón, López y Corujo, 2006).

Entre algunos beneficios de la integración curricular, están:

- Aumenta las interacciones.

- $\quad$ Promueve la participación.

- $\quad$ Estimula la creatividad. 
- Aumenta el interés por investigar y escuchar.

- Genera el diálogo, cuestionamiento y necesidad de asumir actitudes y toma de decisiones.

\section{Figura 1. Integración}

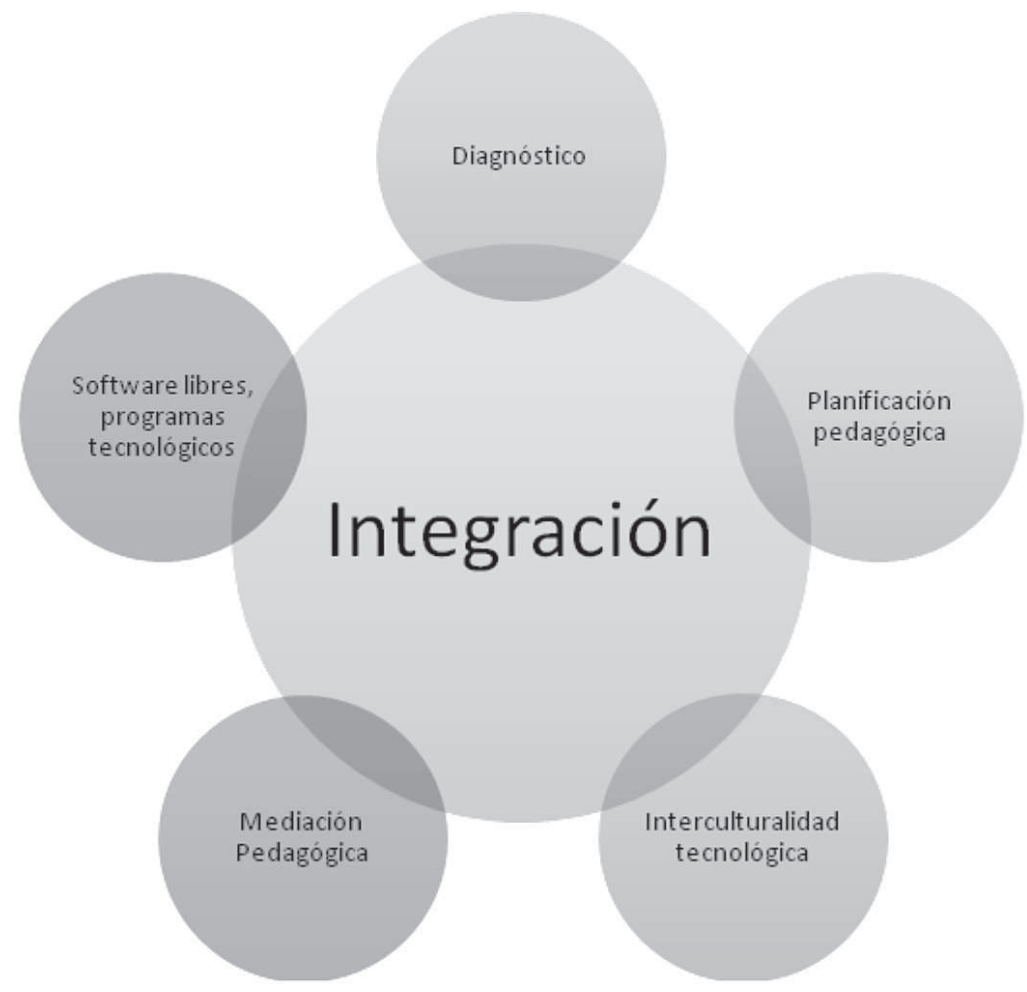

Nota: Propuesta de los (las) investigadores(as)

Por otra parte, Cintrón y Corujo (2006) plantean que es importante desarrollar competencias que faciliten y propicien la integración entre los miembros de un equipo y para lograrlo proponen siete acciones:

\section{Las rutinas de eficiencia}

Básicamente buscan que los miembros de un grupo, de una forma individual, orienten valores, hábitos personales que faciliten la integración y establezcan una micro conducta común sobre la que se construye la integración. Un ejemplo sencillo es el hecho de registrar, de forma detallada, el uso del tiempo de hoy y planear el día siguiente; esto servirá para orientar la eficiencia del grupo; en cambio la acción 
por urgencias, moviéndome en la dirección del menor esfuerzo, dificulta la participación en el equipo.

\section{El placer $y$ disfrute}

Cuando se obtienen logros individuales y comunes y se comparten con los demás participantes, se permite lograr cohesión, sentirse bien emocionalmente $\mathrm{y}$, ante alguna desilusión o presión, evita la desintegración.

\section{La disciplina}

Se refiere a revisar semanalmente las responsabilidades que corresponden a todo el grupo y cómo se logra esto con la responsabilidad y entrega de cada uno y cada una. Esta reflexión permite que cada integrante sienta su responsabilidad al aportar su parte y al apoyarse en las demás personas. De esta manera, se genera un ambiente de confianza.

\section{El descubrimiento}

Es necesario que el grupo comprenda y descubra las habilidades y fortalezas de cada integrante, y que son líderes en la organización en la medida en que tomen parte del proceso creativo. Es impulsar la iniciativa personal, invitando a los integrantes a innovar, a ir más allá de lo conocido.

\section{El servicio}

Los grupos, en este caso las instituciones educativas, necesitan que se trabaje de forma integrada y entender que requiere de los otros y las otras: por encima de los intereses personales debe prevalecer la necesidad de crecer sirviendo, siendo útiles.

\section{Mediación pedagógica utilizando las TIC}

Las tecnologías de la información y comunicación (TIC) siguen en un constante avance, a tal punto que es urgente repensar su función en los procesos de mediación pedagógica; es decir, ¿cómo se están empleando los recursos tecnológicos?, ¿será cómo objetos de aprendizaje?, ¿cómo medios de interacción?, ¿cuál es el uso que se da a la computadora, las tabletas, los celulares inteligentes y otros, en los procesos de enseñanza-aprendizaje? 
Todo lo anterior, conlleva a posicionar al personal docente en un nivel de exigencia en el uso y manejo de las TIC, de tal manera, que esa tarea o responsabilidad por promover el aprendizaje con el estudiantado provoque un impacto en ese proceso de mediar.

Hay que entender que la mediación pedagógica implica una planificación inteligente y cuidadosa, ya que de esto depende la interrelación y los alcances en la construcción del conocimiento. Por lo tanto, un mediador debe ser capaz de promover y orientar el aprendizaje. De esta manera, Cabello y Levis (2007), citando a Gutiérrez, enfatizan que la "mediación pedagógica consiste en la tarea de acompañar y promover el aprendizaje" (p. 242).

Para comprenderlo de una manera más compleja, Fainholc (2004), describe que las mediaciones pedagógicas se encuentran representadas por la acción o actividad, intervención, recurso o material didáctico usados en el hecho educativo para facilitar el proceso de enseñanza y de aprendizaje, por lo que posee carácter relacional. Su fin central es facilitar la intercomunicación entre el estudiantado y los orientadores para favorecer, a través de la intuición y del razonamiento, un acercamiento comprensivo de las ideas a través de los sentidos (Eisner, 1994, p. 16), dentro del horizonte de una educación concebida como participación, creatividad, expresividad y racionalidad.

En este sentido, entre otras tareas, el acto que le compete propiamente al personal docente es el de crear los espacios y aumentar las posibilidades para que el estudiantado desarrolle destrezas y habilidades en el recorrido por aprender, tomando en cuenta que para ello es necesario aplicar estrategias adecuadas que garanticen un verdadero aprendizaje significativo. Se pueden identificar algunas habilidades que el profesor en su tarea de mediar debe contemplar:

- $\quad$ Capacidad de formular preguntas que produzcan conflicto cognitivo.

- Capacidad de generar dudas en el estudiantado, motivándolo a comprender o resolver problemas.

- Motivar al estudiantado al desarrollo del pensamiento.

- Interpretar cuando el estudiantado tiene vacíos o bloqueos que requieren ser apoyados en la construcción de su propio aprendizaje.

- Habilidad para identificar estrategias metacognitivas del estudiantado. (Araya, 2004). 
Sí es importante reconocer que el proceso de mediar la pedagogía no es un hecho aislado en el que el grupo docente sea el único responsable, sino que interfieren diferentes aspectos, entre ellos: el discurso de la mediación, el aprendizaje con el docente, la madurez pedagógica, la cultura, el currículo y la comunicabilidad (Fredez, 2012).

$\mathrm{Al}$ respecto, se puede considerar que mediar no es simplemente dictar información, escribir en una pizarra, repetir información o de contenidos; sino que es un acto complejo que integra al docente, al estudiante, al sistema educativo, a las políticas educativas encargadas del diseño curricular, entre otras.

Hasta aquí se ha tratado de aclarar algunos términos que ayudan a fundamentar esta investigación. Sin embargo, lo que se quiere recalcar es que la mediación pedagógica no se puede aislar del componente tecnológico, también confluyen otros componentes que diferencian la forma de mediar la pedagogía desde lo que se ha conocido como tradicional.

Fainholc (2004) propone que, para profundizar una perspectiva desde la tecnología educativa, se debe sostener el diálogo y la interactividad didáctica provocando y posibilitando, en las mediaciones pedagógicas, que el estudiante ejerza estas funciones de un modo particular en el espacio diseñado para dicha interacción.

Esta interacción a la que se refiere el autor varía en la forma tradicional de aprender, ya que las tecnologías implican nuevas formas de codificar la información, de comprender el lenguaje informático; en otros escenarios trasciende el uso de plataformas virtuales, herramientas didácticas, recursos audiovisuales, que tratan de hacer del aprendizaje una forma diferente, con sentido para la vida, e inclusive hacen del aprendizaje una manera más atractiva y dinámica.

\section{Estrategias para el uso de las TIC en la mediación de los procesos de aprendizaje}

En diversas ocasiones se ha escuchado una frase popular que aduce que la tecnología no es un fin en sí misma, sino un medio. Efectivamente, esto es una realidad que ocurre no solo en este campo, sino en cualquier ámbito de la educación. La didáctica responde, en gran medida, a esta interrogante. Sin embargo, se considera que hoy las tecnologías no son propiamente un medio para el aprendizaje, sino que 
están estrechamente articuladas. Es decir, pasan desde ser un medio para el aprendizaje, a ser parte del aprendizaje.

Tal como se concibió en el apartado anterior, para referirse al término de mediación pedagógica hay que pensar en la relación enseñanza-aprendizaje. Se ha explicado el rol que tiene el profesorado y algunas habilidades que interfieren en ese binomio. Sin embargo, acá interesa mencionar algunas estrategias que permiten la incorporación de las tecnologías en los procesos de mediación, que facilitan y potencian las posibilidades de aprendizaje. Además, es preciso conceptualizar el concepto "aprendizaje" para comprender los términos en que se emplea. Para Araya (2004, p. 2), "la actividad de aprendizaje es una "inter-actividad", un conjunto de acciones culturalmente determinadas y contextualizadas que se llevan a cabo en cooperación con otros".

También, la persona por sí misma, puede aprender a partir de sus conocimientos previos y de su realidad. Vigotsky (1978) distingue dos niveles de aprendizaje:

1. Nivel de desarrollo real (NDR): conjunto de actividades que el sujeto puede hacer por sí mismo, de un modo autónomo, sin la ayuda de los demás.

2. Nivel de desarrollo potencial (NDP): nivel de actividades que podría alcanzar el sujeto con la colaboración y guía de otras personas, es decir, en interacción con los otros.

En el primer nivel, según Vigotsky (1978), la persona no precisamente aprende en compañía de otros, sino que a través de un objeto, una idea, una experiencia personal y puede aprender por sí sola. Eso es muy importante cuando se trabaja con las tecnologías, ya sea de forma virtual o bimodal.

Ahora bien, cuando se recurre al apoyo de las tecnologías en los procesos de formación, se puede decir que esta es considerada un área compleja en la que es necesaria una planificación coordinada, pensada; ante todo es fundamental la selección de los recursos didácticos tecnológicos que garanticen aprendizajes reales y significativos. Precisamente, aunado a esta selección de los recursos, surge una pregunta relevante que viene a responder la interrogante: ¿Cómo incorporar las TIC en la mediación de los procesos de aprendizaje? Es decir, cuáles estrategias didácticas pueden contribuir a mejorar cambios en la forma 
tradicional de impartir las clases, y a propiciar espacios de aprendizajes motivadores, autónomos y, principalmente, generadores de cambios de paradigma del aprender; que permitan pasar de un estado pasivo a un estado activo y crear, en el estudiantado, un aprendizaje más autónomo.

Por ejemplo, si se consideran algunas herramientas didácticas que responden a la interrogante anterior, se puede aplicar el Exe-learning. Todas estas comparten ciertas características comunes, además de que tienen la posibilidad de ser aplicadas en contextos virtuales. Algunas de esas características son herramientas colaborativas y cooperativas, que permiten la interacción según la población meta. En ese sentido, el docente puede aplicarlas para programar sus clases de manera virtual, donde el estudiantado cuenta con el apoyo y las orientaciones de lo que se intenta para orientar el aprendizaje, le permite la posibilidad de aprender a su propio ritmo apoyado con otros recursos tecnológicos de acceso virtual (Araya 2004).

\section{Competencias del docente para la aplicación de las TIC}

La formación profesional abarca todas sus dimensiones, por lo cual toda persona que esté interesada y decidida a incursionar en el campo de la educación debería tomar en cuenta que solo con ese hecho ya tiene una gran responsabilidad. Aún más, actualmente, con estos avances tecnológicos el docente puede desarrollar ciertas habilidades que le conduzcan a ser un profesional con la capacidad de innovar y estar atento a los cambios constantes de la época. Una sociedad del conocimiento y de la información, donde la niñez y la juventud están siendo absorbidas por los constantes avances de la tecnología y son llamados a hacer uso de diferentes herramientas, objetos y equipos que están a su alcance, lo cual ha facilitado, entre otras acciones, su comunicación, su interacción con sus pares y la inserción en una sociedad ya de por sí llamada tecnológica. Como lo menciona Pascual (2012), la aparición de nuevos dispositivos móviles, el abaratamiento de las tarifas de conexión a internet, así como el incremento de redes Wi-Fi gratuitas han provocado un mayor uso de internet en la población y más concretamente en los jóvenes.

Para esta sociedad de niños-jóvenes tecnológicos, se requieren docentes que respondan, profesional y hasta personalmente, con un dominio básico en el manejo de esta variedad de herramientas de las TIC. 
Un docente con posibilidades de avanzar a la par, no solo de las últimas versiones en herramientas digitales, sino en un ambiente donde es indispensable conocer el interés y las inquietudes estudiantiles y acompañarles en el desarrollo del conocimiento.

Alonso (2010), define tratamiento de la información y competencia digital como una de las competencias básicas que deben estar presentes en el currículo educativo. De acuerdo con esto, se necesita un personal docente proactivo, abierto a nuevas posibilidades de aprender, de capacitarse, y de no quedarse rezagado en los conocimientos actuales de esta sociedad digital, para integrarse en los procesos educativos.

Es necesario ir minimizando estos temores que abonan la resistencia al cambio, para enfrentar esta sociedad educativa que en las últimas dos décadas ha visto la incursión de nuevos recursos didácticos tecnológicos ausentes hasta el momento.

Se necesitan educadores y educadoras que comprendan cómo hacer uso de las diferentes herramientas TIC y la aplicación de éstas en el desarrollo de la clase, de actividades del currículo, que propicien nuevos espacios de aprendizaje; quienes a su vez consideren las lecciones más atractivas y más significativas. Como bien cita Salinas (s.f., p. 25): "Los nuevos sistemas de enseñanza configurados alrededor de las telecomunicaciones y las tecnologías interactivas requieren una redefinición de los modelos tradicionales para conducir a un tipo de procesos de enseñanza aprendizaje más flexibles".

Por otra parte, Benavente (2007), plantea que es necesario tener un dominio básico en el manejo de las TIC. Se refiere a ello, indicando que la primera barrera que debe vencerse es la de la competencia tecnológica básica, por parte de docentes, quienes deben comprender el funcionamiento del sistema operativo de su equipo (Windows, Macintosh, Linux) y el uso de las herramientas básicas del sistema operativo como el explorador de archivos, editor de gráficos, papelera de reciclaje, etc.; aprender el uso de programas principales de una "suite" de oficina (procesador de texto, hoja de cálculo, manejador de bases de datos, software de presentaciones); además, debe conocer con propiedad el uso del correo electrónico y de los diferentes navegadores de internet.

Son necesarias: la competencia digital básica, donde los profesionales en educación tengan los conocimientos mínimos, de esta forma, la capacidad de desarrollar actividades mediante el uso de herramientas TIC; procesos de aprendizaje cooperativo, donde el tener un objeto 
móvil en la mano no es señal de dominio de este o de su uso adecuado, más que usarlo por usarlo, es darle un uso adecuado en favor de proceso educativo y de aprendizaje de los estudiantes y del mismo docente. Para ello, es indispensable la formación de los educadores en estas áreas.

Diferentes instituciones ofrecen variedad de cursos, talleres, seminarios y otras actividades, como también los hay en línea, donde la virtualidad ofrece una variedad de ellos y con grandes ventajas para sus usuarios y usuarias: no se puede ni se debe negarse a la inscripción en alguno de estos espacios de capacitación y actualización.

De todo lo anterior, se puede considerar, a manera de resumen, la posición de Bertty (2010), quien menciona las diferentes competencias que un docente o académico debe poseer para el adecuado manejo de TIC. Ellas son:

- $\quad$ Tener una actitud positiva hacia las TIC, instrumento de la cultura actual que conviene saber utilizar y aplicar en muchas actividades domésticas y laborales.

- Conocer los usos de las TIC en el ámbito educativo.

- Conocer el uso de las TIC en el campo de su área de conocimiento.

- Utilizar con destreza las TIC en sus actividades: editor de textos, correo electrónico, navegación por internet, etc.

- $\quad$ Proponer actividades formativas a los alumnos que consideren el uso de TIC.

- $\quad$ Evaluar el uso de las TIC.

\section{Importancia de la formación y actualización docente}

Por muchos años se ha discutido el tema de la pertinencia de la educación frente a los retos que presenta el nuevo siglo. La niñez, la juventud y las personas adultas se enfrentan a una oferta educativa, tanto académica - diurna y nocturna- como técnica. Esta no les ofrece, con propiedad, los instrumentos para desarrollar al máximo sus talentos individuales; su capacidad para enfrentar, con criticidad y creatividad, la velocidad del cambio tecnológico, científico, social, económico, cultural, político, etc.; características dominantes en la nueva era. Tampoco les ofrece las herramientas para aprender a aprender, aprender a pensar (Delors, 1996), aprender a comprender, aprender a vivir juntos y aprender a navegar en la información (Paniagua, 2004). 
Dentro de este panorama, el rol docente es vital, pues le corresponde ser uno de los más importantes actores sociales para enfrentar estos retos, por lo tanto, de su formación y permanente actualización dependerá de que el país pueda enfrentar el tercer milenio con su revolución científico-tecnológica, la globalización del planeta, la conciencia del deterioro del ambiente y la urgencia de hacer sostenible el desarrollo socioeconómico. También, con el descalabro de las teorías que hasta hace muy poco constituyeron la base explícita de las propuestas educativas por la presencia de nuevas alternativas que abren el camino a un nuevo quehacer educativo (Manterola, 1995).

\section{Metodología empleada}

Esta investigación se ubica en el paradigma naturalista, desde un enfoque fenomenológico, debido a que se centra en la experiencia humana y en captar el significado del proceso de actualización desde la experiencia de sus participantes a través de la reflexión.

El lugar donde se realizó la capacitación fue en Nicoya - Nandayure, en las instalaciones del Ministerio de Educación Pública de este lugar.

Participaron dieciocho docentes de escuelas y colegios de Nandayure, quienes fueron convocados por la regional del MEP, institución que brindó los permisos correspondientes. Se formalizó este proceso con una invitación, de parte de la Universidad Nacional, por solicitud de dos estudiantes de la maestría en Administración Educativa (ambos docentes de esa zona), y por el apoyo del Proyecto UNA Experiencia Educativa en Colegios Amigos y Esperanza Joven, de la División de Educología.

El taller Elaboración de recursos didácticos con el apoyo de la tecnológica se llevó a cabo de forma bimodal, 20 horas presenciales y 20 horas virtuales, de manera que se obtuviera un producto y que quienes lograran las 40 horas se hicieron acreedores a un certificado de aprovechamiento, mientras a quienes no las completaron se les entregó certificado de participación.

\section{Instrumentos y técnicas utilizadas}

Se aplicaron entrevistas estructuradas a un grupo focal y cuestionarios a todos los docentes y las docentes, para identificar necesidades de capacitación (diagnóstico); autovaloración y coevaluación del 
desarrollo del taller e interés de sus participantes para generar espacios en próximos talleres de capacitación y actualización.

\section{Población meta}

Las personas que participaron en este taller de capacitación fueron formadores en educación primaria y secundaria, los cuales laboran en instituciones rurales educativas, un total de dieciocho docentes, del cual once de ellos son mujeres y siete son hombres. Sus edades se comprenden entre los 30 y 61 años y se destacan los años de laborar en el sector educativo. Once docentes tienen más de quince años de experiencia, seis de ellos tienen entre once y quince años y una persona oscila entre los cuatro y ocho años de experiencia.

\section{Análisis, discusión e interpretación de los resultados}

A continuación se presentan los resultados de los cuestionarios aplicados en esta investigación.

Tabla 1. Sobre las metas y objetivos del curso

\begin{tabular}{|l|c|c|c|c|c|}
\hline & MUY ALTA & ALTA & MEDIA & BAJA & MUY BAJA \\
\hline $\begin{array}{l}\text { Es relevante para mi } \\
\text { trabajo como docente }\end{array}$ & 18 & & 1 & & \\
\hline Realismo y practicidad & 15 & 2 & & & \\
\hline $\begin{array}{l}\text { Claridad, estructuración en } \\
\text { los objetivos }\end{array}$ & 15 & 2 & & & \\
\hline Claridad en las actividades & 15 & 2 & & & \\
\hline
\end{tabular}

Nota: Elaboración propia de los investigadores, año 2012.

Las expectativas por parte de los participantes, tal y como se muestra en la tabla, se han cumplido en un alto nivel. Es decir, en términos generales, en cada uno de los puntos señalados, el taller propuesto tiene sentido para su profesión. La totalidad coincide en que este taller tiene gran relevancia para su labor docente; de ello se destaca además la practicidad y la realidad para sus vidas.

Esta situación muestra una necesidad evidente en la formación docente: a pesar de los avances en este tema, no precisamente todos son beneficiados; al respecto, hay una gran urgencia por atender en el sistema educativo costarricense. Esta se traduce en capacitar a los 
formadores en la educación formal para que puedan crear cultura en primera instancia y, por su parte, darle el mayor aprovechamiento a los recursos que ofrecen las TIC en el campo educativo.

Tabla 2. Lo que piensa el profesorado participante respecto a los procesos de la mediación pedagógica en el taller impartido

\begin{tabular}{|l|c|c|c|c|c|}
\hline & MUY ALTA & ALTA & MEDIA & BAJA & MUY BAJA \\
\hline Claridad en la exposición & 14 & 3 & & & \\
\hline $\begin{array}{l}\text { Claridad en instrucciones } \\
\text { para realizar las tareas }\end{array}$ & 17 & 1 & & & \\
\hline Capacidad de motivación & 15 & 2 & 1 & & \\
\hline $\begin{array}{l}\text { Dominio de una variedad } \\
\text { de métodos didácticos }\end{array}$ & 15 & 3 & & & \\
\hline Dominio del contenido & 16 & 2 & & & \\
\hline $\begin{array}{l}\text { Capacidad para propiciar la } \\
\text { reflexión }\end{array}$ & 15 & 3 & & & \\
\hline $\begin{array}{l}\text { Facilidad para mantener } \\
\text { relaciones interpersonales, } \\
\text { apertura, saber escuchar }\end{array}$ & 17 & 1 & & & \\
\hline $\begin{array}{l}\text { Capacidad para fomentar } \\
\text { el trabajo en grupo, dar } \\
\text { instrucciones claras, } \\
\text { facilitar el trabajo }\end{array}$ & 17 & 1 & & & \\
\hline $\begin{array}{l}\text { Capacidad para mediar } \\
\text { y resolver situaciones de } \\
\text { conflicto }\end{array}$ & 17 & 1 & & & \\
\hline
\end{tabular}

Nota: Elaboración propia de los investigadores, año 2012.

Tal como se aprecia en la tabla 2, la mediación pedagógica en cualquier proceso que intente hacer que la enseñanza y aprendizaje sean reales, y que logre esa interacción bidireccional entre profesores y estudiantes, responde de la mejor manera. De este modo, cada uno de los puntos que interfirieron en la mediación pedagógica, en términos generales, refleja que hay una satisfacción en la forma que fue mediado el proceso.

En la tabla 2, la mayoría califica la mediación pedagógica del taller en el rango "MUY ALTA", por lo cual se deriva que los procesos de mediación fueron bien aceptados y comprendidos. 
Tabla 3. Calidad de los contenidos abordados en el curso

\begin{tabular}{|l|c|c|l|l|l|}
\hline \multicolumn{1}{|c|}{ CRITERIOS } & MUY ALTA & ALTA & MEDIA & BAJA & MUY BAJA \\
\hline Claridad & 16 & 2 & & & \\
\hline $\begin{array}{l}\text { Sensibilidad de } \\
\text { aplicación práctica }\end{array}$ & 16 & 2 & & & \\
\hline $\begin{array}{l}\text { Se obtuvo un } \\
\text { producto }\end{array}$ & 15 & 3 & & & \\
\hline Estructuración & 15 & 3 & & & \\
\hline
\end{tabular}

Para los docentes participantes, el abordaje de los contenidos del curso cumplió con expectativas muy altas, la estructura del tiempo y la claridad con la que se desarrollaron los temas fue muy clara y de fácil comprensión, de acuerdo con los conocimientos que tenían.

La información presentada en la tabla 4, es el resultado del cuestionario aplicado al grupo focal formado por cuatro docentes seleccionados al azar.

Tabla 4. Opinión del grupo focal

\begin{tabular}{|c|c|c|c|c|}
\hline Preguntas & Sujeto 1 & Sujeto 2 & Sujeto 3 & Sujeto 4 \\
\hline $\begin{array}{l}\text { 1. ¿Considera } \\
\text { que es posible } \\
\text { poner en práctica } \\
\text { lo aprendido } \\
\text { (las TIC) en } \\
\text { los procesos de } \\
\text { mediación en sus } \\
\text { clases? }\end{array}$ & $\begin{array}{l}\text { Es muy difícil } \\
\text { porque se pierde } \\
\text { mucho tiempo } \\
\text { cuando los } \\
\text { grupos son muy } \\
\text { grandes. En } \\
\text { grupos pequeños } \\
\text { puede ser } \\
\text { factible, en mi } \\
\text { caso es limitante. } \\
\text { En lo personal } \\
\text { me dio más } \\
\text { confianza al usar } \\
\text { mi máquina. }\end{array}$ & $\begin{array}{l}\text { Sí, pero es } \\
\text { importante } \\
\text { tener apoyo del } \\
\text { profesor que está } \\
\text { en el laboratorio } \\
\text { de cómputo, para } \\
\text { mí es muy difícil. } \\
\text { Me ayudó a } \\
\text { perder el miedo. }\end{array}$ & $\begin{array}{l}\text { No es posible, } \\
\text { tenemos muchos } \\
\text { problemas de } \\
\text { conectividad } \\
\text { en la zona, mi } \\
\text { colegio queda } \\
\text { muy retirado. } \\
\text { Sin embargo, yo } \\
\text { tengo compu y } \\
\text { con el teléfono } \\
\text { acceso a internet }\end{array}$ & $\begin{array}{l}\text { Claro la unidad } \\
\text { didáctica que } \\
\text { hice la puedo } \\
\text { imprimir para } \\
\text { utilizarla porque } \\
\text { está muy } \\
\text { práctica, muy } \\
\text { motivadora. } \\
\text { Voy a enseñarle } \\
\text { con mayor } \\
\text { seguridad al } \\
\text { estudiantado. }\end{array}$ \\
\hline $\begin{array}{l}\text { 2. ¿Ha recibido } \\
\text { algunas } \\
\text { capacitaciones } \\
\text { sobre el tema de } \\
\text { tecnologías? ¿En } \\
\text { cuál Institución? }\end{array}$ & $\begin{array}{l}\text { Es la primera que } \\
\text { he recibido. }\end{array}$ & \begin{tabular}{|l|} 
Sí en la \\
universidad \\
privada donde \\
estudio me han \\
dado algunas \\
clases de uso de \\
TIC.
\end{tabular} & $\begin{array}{l}\text { Sí, por el MEP, } \\
\text { pero mucha } \\
\text { teoría y poca } \\
\text { práctica. }\end{array}$ & $\begin{array}{l}\text { Sí, pero no había } \\
\text { aprendido nada } \\
\text { útil para utilizar } \\
\text { en el aula. }\end{array}$ \\
\hline
\end{tabular}




\begin{tabular}{|c|c|c|c|c|}
\hline Preguntas & Sujeto 1 & Sujeto 2 & Sujeto 3 & Sujeto 4 \\
\hline $\begin{array}{l}\text { 3. ¿Considera } \\
\text { que es importante } \\
\text { que los docentes } \\
\text { conozcan sobre } \\
\text { como utilizar las } \\
\text { tecnologías? ¿Por } \\
\text { qué? }\end{array}$ & $\begin{array}{l}\text { Claro, es } \\
\text { importante, para } \\
\text { estar a la par de } \\
\text { los estudiantes. }\end{array}$ & $\begin{array}{l}\text { Por supuesto que } \\
\text { sí, debemos estar } \\
\text { actualizados. } \\
\text { Para mejorar } \\
\text { nuestras clases. } \\
\text { Motivar a los } \\
\text { estudiantes. }\end{array}$ & $\begin{array}{l}\text { Sí. Porque me } \\
\text { ayuda a ser mejor } \\
\text { profesional, } \\
\text { porque le ayuda } \\
\text { al estudiante } \\
\text { cuando ingresa a } \\
\text { la universidad. }\end{array}$ & $\begin{array}{l}\text { Sí. Las cosas } \\
\text { en educación } \\
\text { cambian, no } \\
\text { podemos seguir } \\
\text { con lo de hace } 20 \\
\text { años. }\end{array}$ \\
\hline $\begin{array}{l}\text { 4. ¿Le gustaría } \\
\text { participar } \\
\text { en otras } \\
\text { capacitaciones? } \\
\text { ¿En qué temas? }\end{array}$ & $\begin{array}{l}\text { Me gustaría que } \\
\text { no sea esta la } \\
\text { única vez que se } \\
\text { nos brinde esta } \\
\text { oportunidad de } \\
\text { capacitarnos por } \\
\text { parte de la UNA. } \\
\text { Temas que me } \\
\text { interesaría, sobre } \\
\text { el uso de los } \\
\text { correos. }\end{array}$ & $\begin{array}{l}\text { Por supuesto, } \\
\text { espero que } \\
\text { vuelvan a } \\
\text { capacitarnos... } \\
\text { Me gustaría } \\
\text { conocer más } \\
\text { sobre el uso } \\
\text { de celulares } \\
\text { inteligentes. }\end{array}$ & $\begin{array}{l}\text { Sí... } \\
\text { Temas como } \\
\text { software libres. } \\
\text { Uso de correos } \\
\text { electrónicos. }\end{array}$ & $\begin{array}{l}\text { Sí. } \\
\text { Metodología } \\
\text { de enseñanza } \\
\text { de las TIC en } \\
\text { secundaria. } \\
\text { Uso de correos } \\
\text { electrónicos. }\end{array}$ \\
\hline $\begin{array}{l}5 . \text { ¿Cómo se } \\
\text { podría mejorar } \\
\text { este proceso de } \\
\text { capacitación? }\end{array}$ & $\begin{array}{l}\text { Considero que } \\
\text { las metodologías } \\
\text { que utilizaron } \\
\text { al igual que los } \\
\text { temas fueron } \\
\text { excelentes. } \\
\text { Se necesita } \\
\text { más tiempo y } \\
\text { si no pueden } \\
\text { venir podría } \\
\text { usarse más la } \\
\text { virtualidad. }\end{array}$ & $\begin{array}{l}\text { Tal vez hacer una } \\
\text { comunicación } \\
\text { más directa } \\
\text { porque en } \\
\text { algunos } \\
\text { casos no nos } \\
\text { brindaron toda la } \\
\text { información de } \\
\text { los recursos que } \\
\text { se iban a utilizar } \\
\text { en las clases. }\end{array}$ & $\begin{array}{l}\text { Todo estuvo bien. } \\
\text { Me ayudó a tener } \\
\text { más confianza en } \\
\text { las TIC. }\end{array}$ & $\begin{array}{l}\text { Podrían } \\
\text { invitarnos a } \\
\text { cursos que den } \\
\text { en la UNA, y } \\
\text { coordinar con } \\
\text { el MEP para } \\
\text { que podamos } \\
\text { participar y } \\
\text { aprender más. } \\
\text { Muchas gracias } \\
\text { por tomarnos en } \\
\text { cuenta. }\end{array}$ \\
\hline
\end{tabular}

Los participantes y las participantes del grupo focal opinan lo importante de este tipo de capacitaciones y actualizaciones en cuanto a las TIC, sobre todo muestran un interés por aprender y ser cada día mejores docentes para motivar al estudiantado en sus procesos de aprendizaje.

Califican los talleres de forma excelente, y hacen ver lo necesario de la comunicación con las autoridades del MEP, para apoyarlos en su aprendizaje. Valoran lo excelente del trabajo colaborativo aplicado en los talleres.

Se mostraron agradecidos en todo momento por haberlos tomado en cuenta en esta ocasión e insistieron en la necesidad de continuar capacitándose, para perder el miedo a las tecnologías.

Estas opiniones se relacionan con lo que cita Benavente (2007): es necesario conocer y tener un dominio básico en el manejo de las TIC. Se 
refiere a ello indicando: que la primera barrera que debe vencerse es la de la competencia tecnológica básica por parte de las personas docentes.

Por otra parte, según Bertty (2010), una de las diferentes competencias que un docente o académico debe poseer para el adecuado manejo de TIC es su actitud. Al respecto, quienes participaron mostraron una actitud por aprender muy positiva, se evidenciaron sedientos de querer saber cada día más y más. Esto propició un ambiente de aprendizaje placentero para docentes participantes y personal capacitador.

\section{Conclusiones}

Para los docentes y las docentes de la zona de Nandayure, los talleres de las tecnologías fueron muy significativos, cumplieron con las expectativas de aprendizaje, sobre todo la organización de los temas y la calidad de los expositores fue muy clara y concisa. El uso de metodologías innovadoras motivó la participación de las personas que asistieron al taller.

Para ellos, la calidad humana de los docentes capacitadores fue excelente, lo cual facilitó el interés de los participantes; además, los contenidos cumplieron con las expectativas de aprendizaje, situación que motivó a todos a disfrutar de las TIC.

El personal docente de la zona de Nandayure opinó que los materiales elaborados, producto del desarrollo del taller, fueron de mucha utilidad sobre todo les permitió organizar algunos temas para utilizar con el estudiantado en los procesos de mediación pedagógica.

La educación es una gran vocación, somos constructores privilegiados del mundo, tenemos un desafío en nuestras manos y la sociedad actual necesita que compartamos nuestra experiencia y nuestro proceso de aprendiencia: ser reflexivos, renovarnos y actualizarnos constantemente, buscando dar respuesta a las necesidad del estudiantado de la comunidad; en una escuela, un colegio que mire hacia adentro, sin dejar de mirar hacia fuera, con ojos esperanzados en la educación.

\section{Recomendaciones}

Desarrollar talleres que respondan a los requerimientos de una educación de calidad para todos, por esto es necesario promover la profesionalización de docentes en diferentes temáticas y, dentro de estas, las TIC son una urgencia que no se puede dejar por fuera. 
Considerar que las capacitaciones y actualizaciones deben dar cabida a un vacío existente en una población específica según el contexto. Esto permitirá propiciar una salida de la incapacidad que se tiene en ese momento, sobre todo, tener en cuenta el contexto en el que se está desarrollando la capacitación. Para esto se retoma una frase de Morín (1999, p. 45) "Vivimos en un mundo de complejidad creciente y comprensión retardada".

No cabe duda de que, en esta sociedad tecnológica, el personal docente no debe permanecer ajeno a los avances: no hay que rezagarse, porque las TIC son parte del proceso educativo. Por eso nos cuestionamos: ¿será necesario reestructurar el sistema educativo, reformular el papel del docente?

\section{Referencias bibliográficas}

Alonso, M. (2010). Variables del aprendizaje significativo para el desarrollo de las competencias básicas. Editorial Santa Cruz de Tenerife.

Alonso, O. y Zabatta, V. (2012). Estudio de necesidades: Propuesta metodológica para determinar competencias básicas de los docentes en el uso de las TIC. Memorias del Congreso Internacional TIC y Pedagogía. III Edición. Caracas: IUTA.

Araya, F. (2006). Aprendizaje mediado. Recuperado de http://cognitivamediacion. blogspot.com

Avendaño, R. (1988). Una escuela diferente. La Habana: Editorial Pueblo y Educación.

Assman, H. (2002). Placer y ternura en la educación: Hacia una sociedad aprendiente. Madrid: Narcea.

Bertty, J. (2010). Informática educativa. Universidad Popular del Cesar.

Bermúdez, S. y Rodríguez, R. (1996). Teoría y metodología del aprendizaje. La Habana: Editorial Ciencias Sociales.

Betancourt, M. (1997). Pensar y crear: Educar para el cambio. La Habana: Editorial Academia.

Cabello, R. Levis, D. (2007). Medios informáticos en la educación a principios del siglo XXI. Ciudad Autónoma, Buenos Aires Argentina. Prometeo Libros. Recuperado de https://docs.google.com/a/ una.cr/document/d/1T_1qkLvqgTZNOpPVtQkujQ2P0mJT8EQyZKu3I9SCRdw/edit\#)

Cintrón E, López, M. y Corujo, G. (2006). Principios e ideas que fortalecen un currículo integrado e inclusivo. Colombia: Panamericana Formas e Impresos.

Delors, J (1996). La educación encierra un tesoro. UNESCO. Editorial Santillana, Madrid.

Fainholc, B. (2004). El concepto de mediación en la tecnología educativa apropiada y crítica. Recuperado de http://cmapsinternal.ihmc.us/rid=1119466861556_18 04172076_502/educ.ar\%20\%20Educacion\%20y\%20TIC_\%20El\%20concepto $\% 20 \mathrm{de} \% 20$ mediacion $\% 20$ en $\% 201 \mathrm{a} \% 20$ tecnologia $\% 20$ educativa $\% 20$ apropiada $\% 20 \% 20$ critica.pdf 
Fredes, C. (2012). Pedagogía I y II. Recuperado de: http://www.slideshare.net/ cintiafredes/mediacin-pedaggica-13521789.

Garibay, G. (1996). Universidad y comunidad. Revista Universitaria 2000(2),

Gutiérrez, A. (2007). Integración curricular de las TIC y educación para los medios en la sociedad del conocimiento. Revista Iberoamericana de Educación, 45. Recuperado de http://www.rieoei.org/rie45a06.htm

Gutiérrez, F. y Prieto, D. (1999). La mediación pedagógica: Apuntes para una educación a distancia alternativa (6ta Ed.). Buenos Aires: Ciccus - La Crujía.

Malagón, J. (2009). Mediaciones sociales. La Mediación Pedagógica. NA 5, II semestre pp. 175-180. ISSN electrónico: 1989-0494.

Manterola A. y Carlos, F. (1995). La formación docente: Un reto imprescindible. Planiuc, 14, (21), 46-53.

Mudrik, A. (1991). La educación y la enseñanza: Una mirada al futuro. Moscú: Editorial Progreso.

Paniagua, M. (2004) La formación y la actualización de los docentes: Herramientas para el cambio en educación. Recuperado de http://www.cedal.org/docus/ educ01.pdf

Pérez, G. (1996). Metodología de la investigación educativa. La Habana: Editorial Pueblo y Educación.

Salinas, J. (s. f.). Cambios metodológicos con las TIC. Estrategias didácticas y entornos virtuales de enseñanza-aprendizaje. Universidad de las Islas Baleares. Editorial Universidad.

Salmerón, M. (2011). La integración de las nuevas tecnologías en los centros educativos. EFDeportes.com, Revista Digital, 16(156), 89-95.

Santamaría, M (2012). Innovación docente universitaria en entornos de aprendizaje enriquecidos. I Jornadas Internacionales de Innovación Docente Universitaria en Entornos de Aprendizaje Enriquecidos. Libro de actas: UNED. México.

Vigostky, L..S. (1978). Pensamiento y Lenguaje. Editorial Buenos Aires. 